\title{
Reviving resuscitation skills: Non-invasive ventilator training for ward nurses
}

Zaffer Khan Cusi Malik ${ }^{1}$, MNSc, NP Community Health of Older People (CHOPI); NP, Emergency Department

${ }^{1}$ Capital \& Coast District Health Board, Wellington, Aotearoa New Zealand Corresponding Author: zaffer.malik@ccdhb.org.nz

\author{
Citation \\ Malik, Z. (2021). Reviving resuscitation skills: \\ Non-invasive ventilator training for ward \\ nurses. Nursing Praxis in Aotearoa New Zealand, \\ $37(3), 22-24$. \\ https://doi.org.10.36951/27034542.2021.029
}

\begin{abstract}
As part of the pro-active response in anticipation of a possible outbreak of COVID-19 in Aotearoa New Zealand, ward nurses at Wellington Regional Hospital were requested by senior nursing leadership to participate and engage in non-invasive ventilation training. The objective of this education was to allow adequate response and provision of lifesaving non-invasive ventilation to critically unwell patients. Identifying potential nurses and ascertaining the amount of training and resources involved in mass training was critical to the success of the programme. The variation in ventilation equipment was a significant detail that had contributed to the mounting challenge of addressing the lack of both human and technological resources. This reflection piece describes the education initiative and the circumstances and practicalities of creating, teaching, and training advanced skills (non-invasive ventilation education) to ward nurses with limited respiratory experience.
\end{abstract}

Keywords: COVID-19; intensive care; non-invasive ventilation; nursing

\section{Background}

The COVID-19 pandemic continues to threaten not only the health of people worldwide but has also pushed the healthcare systems globally to the brink of collapse. The most concerning complication of COVID-19 is acute hypoxaemic respiratory failure; a condition that usually requires mechanical ventilation (Wilcox, 2020). Internationally, there is a search to find affordable and practical solutions to treat, manage and care for patients amidst the global shortage in intensive care unit (ICU) beds and ventilator supply (Andellini, et al., 2020).

Non-invasive ventilation (NIV) is an important strategy in COVID-19 management, particularly in countries where there are limited intensive care resources (Bertaina, et al., 2021). Studies have shown that the use of NIV on patients in the early stages of COVID-19 infection potentially avoids the need for intubation and mechanical ventilation (Menzella, et al., 2021).

\section{The Aotearoa New Zealand problem}

Aotearoa New Zealand has limited ICU resources and a wave of COVID-19 patients could have catastrophic effects. As of the 29th of April 2020, Aotearoa New Zealand had a ventilator capacity of 334, and an ICU capacity of 358. Capital and Coast District Health Board (CCDHB) had a ventilator capacity of 37 and an ICU capacity of 31 (Ministry of Health - New Zealand, 2020).

\section{The initiative}

In anticipation of the potentially devastating effect COVID-19 can have on the health system, CCDHB created capability to temporarily increase the medical ward's high-dependency bay (HDB) capacity from four beds to eight beds, with the potential to operate beyond the bed limit dependent on patient requirements. This initiative resulted in the need for an increased number of ward nurses to deliver NIV therapy effectively and safely to affected patients. The objective was to upskill ward nurses to allow adequate provision of timely, lifesaving NIV for unwell patients, improving their health outcomes, 
should the need exceed Wellington Regional Hospital's current capacity. This planning has set a precedent for future pandemic planning that would involve NIV-trained staff.

Due to the overwhelming workload related to the pandemic planning i.e., development of ward-specific COVID-19 protocols, it had been a challenge to get the right people - subject matter experts in the same room. As a nurse practitioner, i worked alongside other colleagues comprising of nurse educators from the medical wards to develop, organise, and facilitate a study day repeated in 10 sessions to upskill identified nursing staff. The study day included both a theoretical and practical module.

One of the initial and significant steps was to gather current training records to ascertain nurses who were currently trained and certified in NIV and to identify potential nurses who may be eligible for NIV certification. The group decided that it would be advantageous if we upskilled a group of nurses who fitted the following criteria: confident and competent in caring for patients with respiratory conditions; previous experience in a medical ward; preferably with previous experience or knowledge on how and when to initiate and control NIV; nurses who were low risk in the event of contracting COVID-19; i.e., age $<65$ years, not pregnant, and not immunocompromised.

We were transparent and realistic with our expectations from the beginning knowing that nurses who were recently trained in NIV would not be experts in the field. Rather, our aim was to increase their awareness and knowledge about NIV therapy and to build confidence and competence over time.

We organised with charge nurse managers to release identified staff for a full-day training followed by an orientation shift in the High Dependency Bay as staffing levels allowed. It was a challenge to plan and undertake the study day, as staffing constraints at times made it arduous to release staff from clinical shifts. Finding a suitable venue to hold training was difficult as we had to ensure that the venue allowed for social distancing, while still promoting collaboration and the feeling of togetherness. Limiting the numbers of attendees meant that the team had to run the sessions more frequently to achieve our goal of training potential nurses.

Developing the programme for the training days was demanding as we had to ensure that the topics presented were relevant, interesting, and that the content was easily understood. The instructors were inspired to teach as they were able to share knowledge and hone the nurses' skills in NIV. The training days had a specific focus on respiratory physiology, respiratory assessment, NIV, and blood gas interpretation. The practical aspect covered the set-up, use, troubleshooting and management of the NIV equipment.

An unexpected challenge resulted from the introduction of new ventilators dissimilar to those currently in use at Wellington Regional Hospital. Orientation to the new ventilators meant that we had to train everyone including ourselves and staff who were deemed as NIV-certified, to familiarise with the new set-up, updates, general operation, cleaning, storage, and maintenance. The new ventilators operate using different pressure measurements from what we currently utilise. The implication is that there is a potential to administer the wrong pressures if staff are not able to decipher or convert the pressure readings from one ventilator to the other. To mitigate the risks, we invested in more resources, energy, and time being allocated to the practical training component compared to what was originally planned.

One of the many things that the Nurse Educators had to prioritise was being present and readily available clinically to empower and educate nurses. Taking advantage of every opportunity to teach has been a key agenda. We wanted nurses to feel supported and enjoy the learning opportunity; to view the situation as an opportunity to excel rather than a challenge to overcome.

\section{Sustaining change}

Senior nurses were allocated the role of "NIV champion". The purpose of this designation was to help in delivering bedside clinical support to their colleagues when required and assisting the Nurse Educators to educate and assess competency and identify training gaps in the ward. To date we have trained an additional 93 nurses, growing the total number of NIV-trained nurses from 46 to 139; a $202 \%$ increase. Most of the trainees will continue to require supervision in the use of NIV, and ongoing support to develop their confidence and competence. However, we consider this a milestone.

Sustainability is an ongoing challenge. Nurse educators need to maintain a robust system for 
administration, forward planning, organisation, record keeping, and provision of accessible training materials to keep traction and momentum. Protected management time to achieve these goals should be embedded in the ongoing pandemic planning. Feedback from the participants after the day's programme had been instrumental in ensuring the training is relevant and has a positive practice impact for everyone involved.

\section{Recommendation}

An NIV steering group should be officially established in our organisation. The development of NIV elearning and training packages should be prioritised and supported by capability and development to promote ongoing, self-initiated learning.

The ongoing global outbreak of COVID-19 continues to threaten the already fragile state of the Aotearoa New Zealand healthcare system. Despite constraints, nurses have consistently shown the world what resilience looks like. Preparedness is key to be able to address ongoing and evolving issues. Having a sustainable organisational, and preferably national training programme for NIV, would ensure that patients receive efficient, effective, and quality care.

\section{References}

Andellini, M., De Santis, S., Nocchi, F., Bassanelli, E., Pecchia, L., \& Ritrovato, M. (2020). Clinical needs and technical requirements for ventilators for COVID-19 treatment critical patients: An evidence-based comparison for adult and pediatric age. Health \& Technology, 10, 1403-141. https://doi.org/10.1007\%2Fs12553-020-00467-w
Bertaina, M., Nunez-Gil, I., Franchin, L., Rozas, I., ArroyoEspliguero, R., Viana-Llamas, M., Romero, R., Maroun, C., Uribarri, A., Becerra-Muñoz, V., Huang, J., Alfonso, E., Marmol-Mosquera, F., Ugo, F., Cerrato, E.,

Fernandez-Presa, L., Roubin, S., Guzman, G., Gonzalez, A., ... Estrada, V. (2021). Non-invasive ventilation for SARS-CoV-2 acute respiratory failure: A subanalysis from the HOPE COVID-19 registry. Emergency Medicine Journal, 38, 359-365. https://doi.org/10.1136/emermed-2020210411https://emj.bmj.com/content/38/5/359 Menzella, F., Barbieri, C., Fontana, M., Scelfo, C., Castagnetti, C., Ghidoni, G., Ruggiero, P., Livrieri, F., Piro, R., Ghidorsi, L., Montanari, G., Gibelline, G., Casalini, E., Falco, F., Catellani, C., \& Facciolongo, N. (2021). Effectiveness of noninvasive ventilation in COVID-19 related-acute respiratory distress syndrome. Clinical Respiratory Journal, 15, 779-787. https://doi.org/10.1111/crj.13361

Ministry of Health New Zealand. (2020). Ventilators and ICU bed capacity. Author. https://www.health.govt.nz/system/files/documents Lpages/ventilators and icu capacity 11 may 2020.pd $\underline{\mathrm{f}}$

Wilcox, S. (2020). Management of respiratory failure due to covid-19. British Medical Journal, 369. https://doi.org/10.1136/bmj.m1786

\section{Funding: None}

Conflict of interest: None 